\title{
LOS RETOS DE LA HUMANIDAD ANTE LA PERSPECTIVA DE 2050 Y LOS BOSQUES
}

\author{
Eduardo Rojas Briales
}

Subdirector general, Responsable del Departamento Forestal, FAO. Viale delle Terme di Caracalla. ROMA (Italia). Correo electrónico: eduardo.rojas@fao.org

\section{Resumen}

Se analizan los principales retos globales identificados en la Cumbre Río+20 (alimentación, energía y cambio climático) y se desarrollan las correspondientes respuestas. Seguidamente se discute el rol de los bosques en este contexto. Se avanza cual podría ser el anclaje de los bosques en los objetivos de desarrollo sostenible que se aprobarán en 2015 como substitutos a los objetivos de desarrollo del milenio. Se recuerda el legado forestal del principio de sostenibilidad definido en 1713 por primera vez por Carlowitz. Finalmente se analizan temas forestales emergentes como los bosques semi-naturales, los bosques secos, el régimen forestal internacional, la gobernanza forestal y la comunicación forestal para finalizar identificando 2015 como un año clave en la agenda internacional.

Palabras clave: Río+20, Objetivos de desarrollo sostenible, Bosques, Sostenibilidad, Régimen forestal internacional

\begin{abstract}
The key global challenges as identified during the Rio+20 Summit (food, energy and climate change) are analyzed and correspondent answers developed. The role of forests in this context is discussed. The possible accommodation of forests in the sustainable development goals to be approved in 2015 as successors of the present millennium development goals is analyzed. Sustainability as a forest cultural legacy defined first by Carlowitz in 1713 is highlighted. Finally emerging forest issues are discussed like the role of semi-natural and dry forests, forests in the international forest regime, forest governance and forest communication. 2015 is presented as a key international year where several crucial international processes coincide.
\end{abstract}

Keywords: Rio+20, Sustainable development goals, Forests, Sustainability, International forest regime

\section{LOS RETOS DE LA HUMANIDAD IDENTIFICADOS EN LA CUMBRE RÍO+20}

En junio de 2012 se celebró la Cumbre Río+20 con el objetivo de celebrar los 20 años de la primera cumbre de Río en 1992 y los 40 de la primera cumbre ambiental global celebrada en Estocolmo en 1972 pero también de fijar la hoja de ruta de la Humanidad con la perspectiva en 2050. El documento "El futuro que queremos" (ONU, 2012a) identifica los principales retos de la Humanidad y aporta claves fundamentales para abordarlos.

Los 7 temas principales escogidos fueron empleo, desastres, alimentación, energía, agua, 
ciudades y océanos. No obstante muchos otros temas están estrechamente relacionados con estos 7 ámbitos recibiendo considerable atención en el documento: cambio climático, suelo, biodiversidad, economía verde, salud o los bosques. La naturaleza común a estos retos es precisamente su carácter transversal y sus considerables interrelaciones.

La ausencia explícita de los bosques se debe, entre otras razones, al considerable fracaso que supuso el intento de convertirlos en sujetos de la cuarta convención en la Cumbre de Río de Janeiro de 1992 (Pons, 2004). No obstante, cabe recordar que durante los Diálogos de Río con la sociedad civil organizados justo antes de la cumbre, la segunda medida más votada por sus participantes fue precisamente la restauración forestal.

Si tuviésemos que resumir en 3 los principales retos de Río+20 serían alimentación, energía y cambio climático condicionados por cambios demográficos (urbanización, envejecimiento, migraciones, progreso socioeconómico) (Figura 1). El principal reto proviene no tanto del crecimiento demográfico estricto previsto hasta 2050 donde se espera que la población mundial pase de 7.000 a 9.000 millones de habitantes, como mucho más la emergencia de las clases medias en los países emergentes a la vez que éstos se extienden entre los actuales países en vías de desarrollo generalizándose el modo de vida occidental.

En el ámbito de la alimentación, se espera un crecimiento de la demanda del $60 \%$ debido al considerable aumento de la demanda de proteína animal y la correspondiente inducida de cereal (pienso) (FAO, 2012a). Paralelamente aumentan los problemas de obesidad debido a una alimentación desequilibrada mientras que las grasas y azúcar han sido durante la segunda crisis alimentaria de 2011 los productos que más han incrementado su precio (FAO, 2014).

Es cierto que África alberga un considerable potencial agrícola inexplotado pero además de otras restricciones (infraestructuras, tenencia), es el continente potencialmente más afectado por el cambio climático.

La bioenergía puede ser clave en la lucha contra el cambio climático pero a corto plazo puede incrementar el precio del cereal o incluso acelerar la deforestación. Se trata de una situación paradójica que recuerda a la del ferrocarril en el siglo XIX donde su construcción comportó a corto plazo una considerable demanda de madera para traviesas si bien a medio-largo plazo contribuyó decisivamente a la movilidad de los combustibles fósiles reduciendo así la presión sobre los bosques.

Se puede concluir que los retos planteados son en sí positivos - no hay mal que por bien no venga - dado que proceden de un intenso proceso que está sacando a millones de seres humanos de la pobreza gracias a la emergencia económica acompañada de políticas sociales decisivas. Estos retos están profundamente interrelacionados y carece de todo sentido plantear abordarlos de forma aislada (Figura 2). Su carácter global

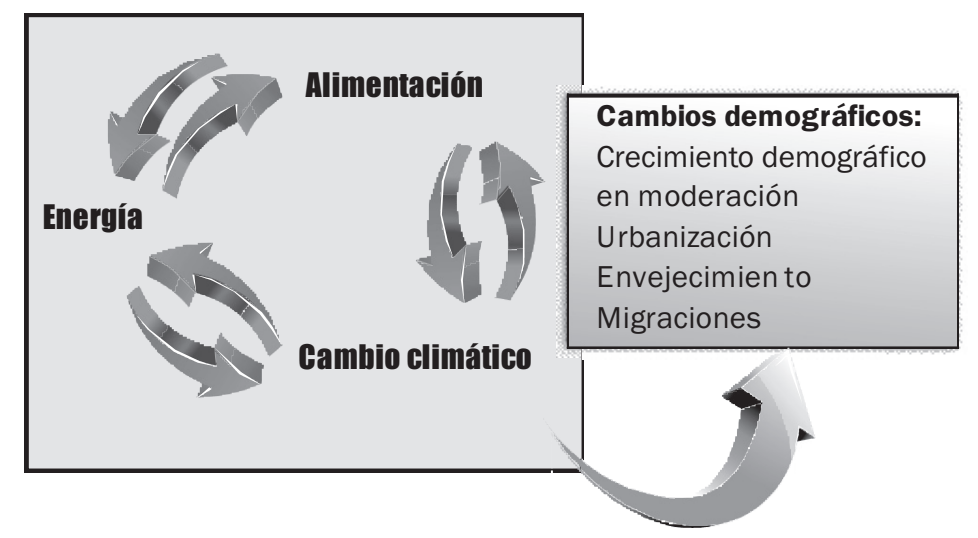

Figura 1. Resumen de los 3 principales retos de Río+20 y su interrelación 
Rio +20 declaration $m$ apping and FAO

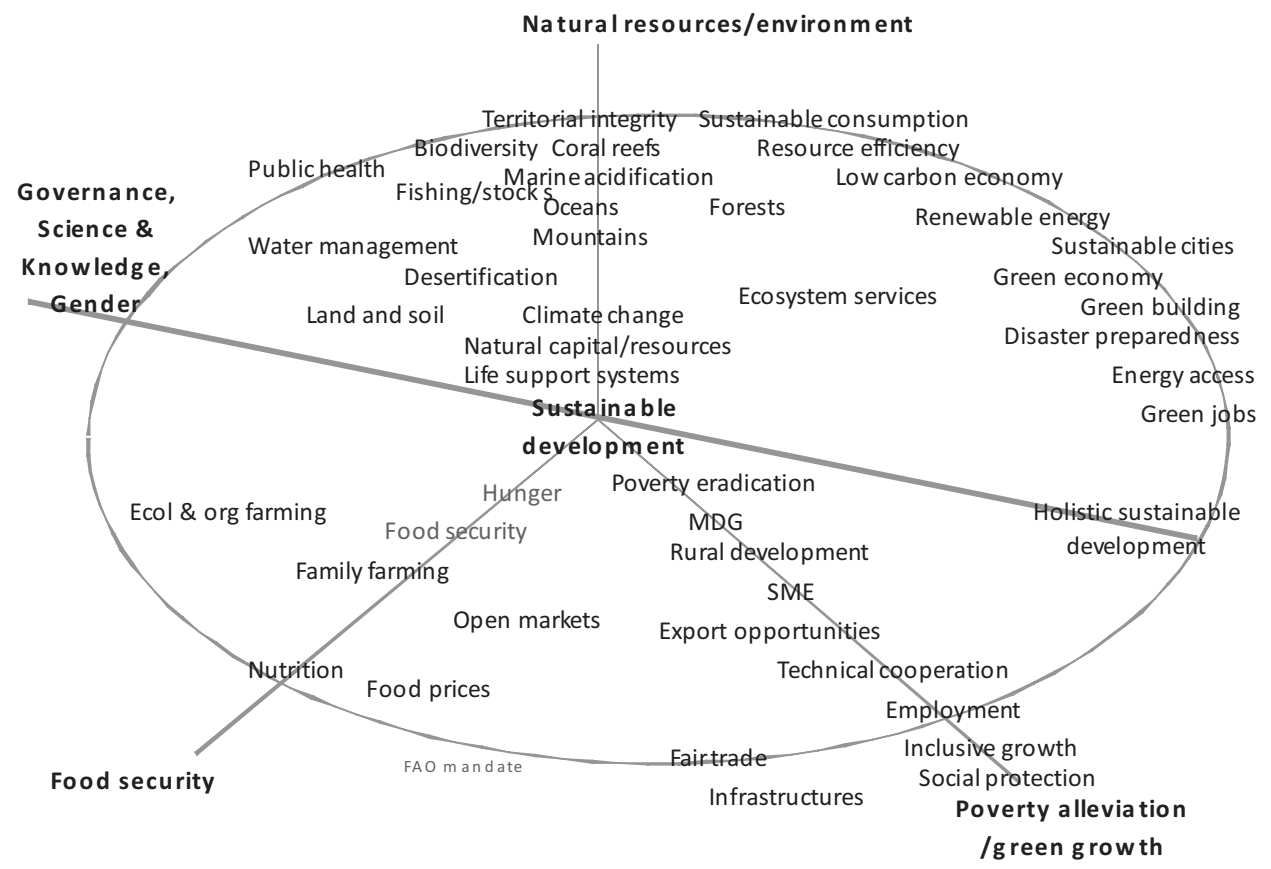

Figura 2. Ejercicio de visualización de los conceptos substantivos incluidos en el Documento de partida de "El futuro que queremos (Río+20)” en relación con el mandato de la FAO y sus 3 objetivos globales

emplaza al único mecanismo de gobernanza existente de escala global, las Naciones Unidas, a tener un rol central y clave.

\section{LAS RESPUESTAS ANTE LOS RETOS DE RÍO+20}

Las respuestas ante los retos planteados podrían resumirse en la siguientes 5:

- Avanzar hacia una ordenación optimizada del territorio rural que incluya su potencial productivo, valor añadido, empleo, reducción del riesgo de desastres, ciclo hídrico, protección del suelo, biodiversidad y valor del paisaje. La protección de los mejores suelos agrícolas ante el avance urbano e infraestructuras es clave, especialmente en Asia.

- Avanzar hacia una tenencia de la tierra más justa, clara y eficaz evitando el acaparamiento de tierras, el predominio formal de la propiedad del estado formal, la duplicidad de regímenes de propiedad contradictorios y la devolución de los derechos de gestión (y propiedad limitada) a las comunidades indígenas o locales ya sea de forma colectiva o individual (FAO, 2012b, 2013).

- Acompañar el proceso de urbanización para evitar un éxodo rural anárquico manteniendo una mínima población viable en el sentido productivo basada en explotaciones medias y un tejido productivo formado por PYMES a la vez que se aseguran servicios públicos equiparables a las zonas urbanas y costeras. Cabe recordar que la agricultura de subsistencia no ha permitido sacar a las poblaciones de la miseria ni ha asegurado un uso sostenible de los recursos.

- Alcanzar en 2015 un acuerdo de cambio climático global ambicioso que permita movilizar todo el potencial del Planeta en las 4 partes de la ecuación:

- reforzando los sumideros vegetales (los únicos gestionables) reduciendo la defo- 
restación y acelerando la restauración forestal (3/4 de los GEI son $\mathrm{CO}_{2}$, la vegetación y el suelo retienen tanto $\mathrm{C}$ como la atmósfera, FAO, 2010)

- reduciendo el consumo energético de forma decidida (transporte público)

- substituyendo las energías fósiles por renovables biológicas (biomasa) o físicas (solar, eólica, mar). En el caso de la biomasa mediante un uso eficiente de los recursos existentes optimizando su función de substitución (uso térmico) allí donde existan recursos adicionales sostenibles (Tabla 1)

- substituyendo materias primas de alto consumo energético por otras de menor, sobre todo renovables (madera en vez de cemento o hierro), especialmente en la construcción

- Se hace necesario modificar los patrones alimenticios aumentando la proporción de verduras, frutas y proteína no cárnica (pescado, insectos) y reduciendo las grasas, azúcares y proteína animal. La ganadería intensiva provoca un aumento sobreproporcional de demanda de cereal para pienso y fuertes emisiones de metano (GEI). La OMS insiste en ir hacia unos hábitos alimenticios y de forma de vida (ejercicio) más saludables reorientando la prioridad de la función pública de la provisión de asistencia sanitaria (sanidad) a asegurar la salud integral de la población.

En definitiva, solo una acción concertada y global será capaz de alcanzar la suficiente masa crítica para poder superar estos retos tan profundamente interrelacionados.

\section{EL ROL DE LOS BOSQUES}

La ordenación del territorio propuesta anteriormente tiene un rol preeminente en la lucha contra la deforestación y contribuirá además a hacer más evidente el rol de los bosques y especialmente de la restauración forestal. El Global Partnership on Forest Landscape Restoration (GPFLR, 2014) ha identificado más de 1.000.000.000 ha disponibles para la restauración forestal sin afectar a la seguridad alimentaria lo que elevaría la cobertura forestal global del actual $31 \%$ al $39 \%$, en parte mediante sistemas agro-forestales o la conducción de los procesos de restauración forestal espontáneos, especialmente activos en zonas con una mínima humedad o de montaña. Todo ello permitirá reorientar REDD+ más allá de la reducción de la deforestación a su reversión confiriéndole un enfoque proactivo que honre el sentido original del + .

\begin{tabular}{l|c|}
\hline Preindustrial $\mathrm{CO} 2$ & $2029 \mathrm{GtCO}$ \\
\hline Increase of the $\mathrm{CO}$ concentration to present & $+767 \mathrm{GtCO}$ \\
\hline Emissions -2050-60\% (70\%) & $+728 \mathrm{GtCO}$ \\
\hline Emissions 2050-2150 -80\% (20\%) & $+443 \mathrm{GtCO}$ \\
\hline $\begin{array}{l}\text { Net-Afforestation 2010-2060 500 M ha grown up } \\
\text { to 2150 (10 M ha/a) }\end{array}$ & $-297 \mathrm{GtCO2}$ \\
\hline $\begin{array}{l}\text { Increased stocks existing forests up to 2150 } \\
\text { (+20\%) }\end{array}$ & $-478 \mathrm{GtCO2}$ \\
\hline $\begin{array}{l}\text { Balance } \\
\text { Marine sequestration(8,3 GtCO2) GtCO2/a) 2010- }\end{array}$ & \\
\hline D15n & $3193 \mathrm{GtCO}$ (+14\% 2010) \\
\hline Balance & $-1163 \mathrm{GtCO2}$ \\
\hline
\end{tabular}

Tabla 1. Escenario a largo plazo capaz de reconducir la concentración de CO2 atmosférico a los niveles preindustriales 
Se hace igualmente necesario abordar los necesarios reajustes en la tenencia de la tierra incluidos los bosques devolviendo el protagonismo a los agentes locales estableciendo marcos seguros y fiables, la asunción progresiva de responsabilidades y la construcción de capacidades auto-organizativas (Cooperativas, asociaciones).

Debe reconocerse el rol estratégico de la función productiva que aunque en muchos casos por sí sola no justifique la actuación pública permite movilizar unos recursos substantivos que difícilmente estarían disponibles en el ámbito público y, en todo caso, con costes de transacción considerables. Entre las opciones más inmediatas destacarían:

- la utilización de toda la bioenergía disponible de forma óptima (térmica y cogeneración) optimizando el valor añadido. En España un aumento del actual $40 \%$ al $60 \%$ del ratio aprovechamiento/crecimiento comportaría un ahorro $3.000 \mathrm{M€}(0,3 \% \mathrm{PIB})$ en costes energéticos así como una reducción $10 \%$ importaciones petróleo (ROJAS, 2011)

- la substitución en la construcción del cemento, hierro, aluminio y plásticos por madera, bambú y corcho en todos los casos técnicamente posibles.

Otro aspecto clave, sobre todo en países en vías de desarrollo se centraría en la movilización de todo el potencial de los bosques en su contribución a la seguridad alimentaria ya sea directa (semillas, frutas, miel, carne, pescado, insectos, hojas, setas, etc.) como indirecta (polinización, leña, forraje para ganado en épocas secas) y servicios ambientales claves para la agricultura y la pesca (regulación del ciclo hídrico, protección del suelo).

Es igualmente importante visualizar la aportación de los bosques al desarrollo humano, especialmente en el ámbito del desarrollo rural y la lucha contra la pobreza. Una forma de visualizar podría consistir en elaborar mapas que recojan la superficie de un país donde los bosques constituyen el primer o segundo motor económico local o que supere un umbral determinado $(25 \%)$.

Finalmente se hace necesario superar debates estériles que únicamente se pueden explicar por luchas corporativas de poder y no en evidencias. Ante la intensidad de las demandas sociales $\mathrm{y}$ retos planteados urge superar el segregacionismo funcional y reforzar la integración de las funciones, especialmente de la preservación de la biodiversidad.

\section{LOS OBJETIVOS DE DESARROLLO SOSTENIBLE}

En 2015 finalizan los actuales Objetivos de Desarrollo del Milenio orientados como su nombre indica al desarrollo y por lo tanto solo aplicables a una parte del planeta. Tal y como critica "El futuro que queremos" la experiencia desde la Cumbre de Río de 1992 coincide en señalar que los tres pilares que conforman el desarrollo sostenible han sido tratados excesivamente de forma aislada también en los Objetivos de Desarrollo Sostenible (ODS, ONU, 2014). Por ello, en Río+20 se decidió establecer para la agenda post 2015 unos nuevos ODS que fuesen de cobertura universal e integrasen los tres pilares de forma equilibrada.

A modo de ejemplo, los bosques aparecían en los objetivos de desarrollo del milenio solo bajo medio ambiente y de forma negativa (deforestación).

El marco dado para los ODS debe ser además de universal, reflejar una aspiración colectiva, de fácil comunicación y limitados en número. En todo caso no debe haber más que capítulos substantivos en el documento "El futuro que queremos".

Es evidente que los bosques contribuyen de forma decisiva a otros ámbitos (energía, cambio climático, alimentación, desarrollo rural, lucha contra la pobreza, agua, suelo, biodiversidad, energía verde, salud, entre otros). Un enfoque limitado a lo que los bosques contribuyen a otros sectores comporta el riesgo de obviar dos elementos clave. En primer lugar, los necesarios recursos compensatorios que actúen como retorno económico para hacer viable la compleja y costosa gestión forestal multifuncional necesaria para asegurar las demandas sociales y las inversiones requeridas para restaurar los bosques y ponerlos en condiciones de responder a ellas (Figura 3). En segundo lugar, los mecanismos de gobernanza que diriman en cada momento la capacidad de carga de los recursos forestales y entre intereses contrapuestos. En definitiva, impediría un enfoque congruente, consistente y viable. Focalizar desproporcionadamente solo 


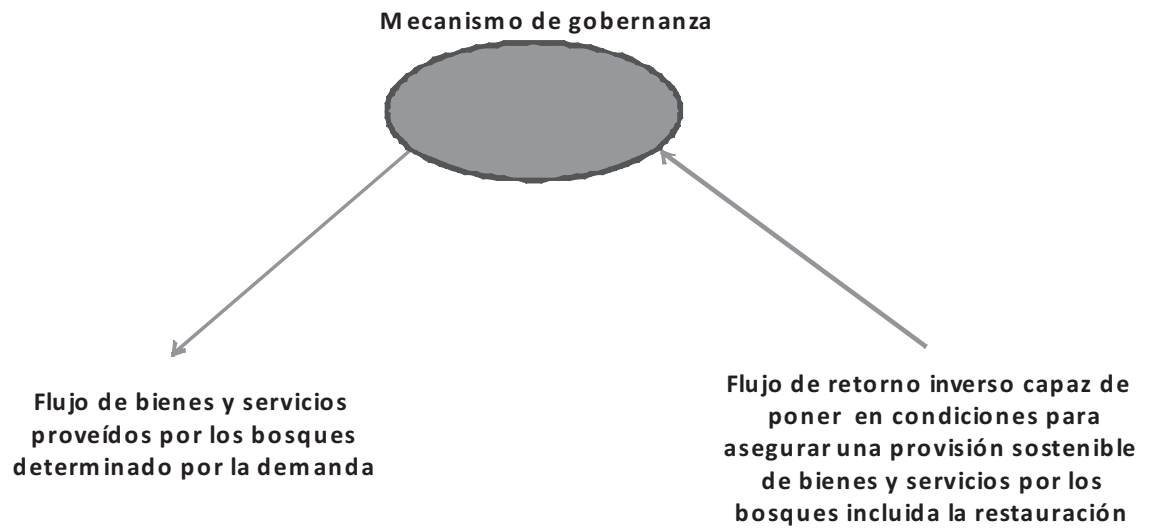

Figura 3. Elementos clave de un posible objetivo de desarrollo sostenible forestal

en aquello que los bosques pueden aportar comportaría el riesgo de repetir lo que se podría denominar el síndrome femenino en las sociedades clásicas: anteponer los servicios aportados en términos de cuidado niños, ancianos, alimentación y el hogar sin considerar la sostenibilidad y equidad de las cargas.

Otro aspecto a considerar es que en el caso de un reducido número de ODS -el objetivo es no superior a 10 - el correspondiente proceso puede ser muy complejo dado que los posibles ámbitos relacionados ya estén incluidos en otros ODS como la agricultura (alimentación) o su inclusión esté aún abierta (biodiversidad y pesca pendientes de la solución que se dé a los océanos), agua con un peso importante del uso urbano, entre otros. Todo ello comporta el riesgo que un ODS de recursos naturales como ha sido propuesto puede quedar circunscrito a un ODS residual formado de los retales que no encajan en otros ODS.

Considerando que en el proceso han caído las montañas pese a disponer de un capítulo substantivo en "El futuro que queremos" y que la FAO es también la agencia responsable del sistema ONU de este ámbito sería factible considerar un ODS de bosques y montañas junto a los demás usos extensivos como matorral, desiertos, zonas húmedas, etc. Actualmente $\left(1^{\text {er }}\right.$ trimestre de 2015) la estructura de ODS está prácticamente ultimada, con un total de 17 , quedando incluido en el ODS15 junto a la biodiversidad, ecosistemas y montañas.

\section{LA SOSTENIBILIDAD, UNA HERENCIA CULTURAL FORESTAL}

En 2013 se celebran los 300 años de la publicación de CARLOWITZ (1713) donde se describe por primera vez la definición de sostenibilidad definida entonces como persistencia lo que a su vez supuso la semilla de la ciencia y consecuentemente gestión forestal profesionalizada (Figura 4). Durante 2013 se han sucedido las celebraciones de esa efeméride que constituye una substantiva contribución forestal al desarrollo de la Humanidad y una importantísima aunque poco reconocida contribución de la Ilustración (RoJAs, 2014).

La primera definición se centra en el eje vertical (continuidad del recurso y sus rentas) pero a medida que las demandas funcionales van apareciendo, va consolidándose el concepto de gestión multifuncional (Engenieurs des Eaux et Fôret, uso recreativo en paralelo a la aparición de los vehículos utilitarios, biodiversidad, cambio climático en paralelo al progreso científico).

Bruntland en el informe "Nuestro futuro común" (ONU, 1987) describe el principio de sostenibilidad reconociendose en todo momento (VON WEIZSÄCKER, 1997) su obvio origen forestal, como queda patente también por la continuidad termonológica tanto en alemán (Nachhaltigkeit) como francés (durabilité), los primeros que usaron la definición de Carlowitz.

Tanto la progresiva ampliación del principio de la persistencia original mediante la multifuncionalidad, como la soistenibilidad de Bruntland 


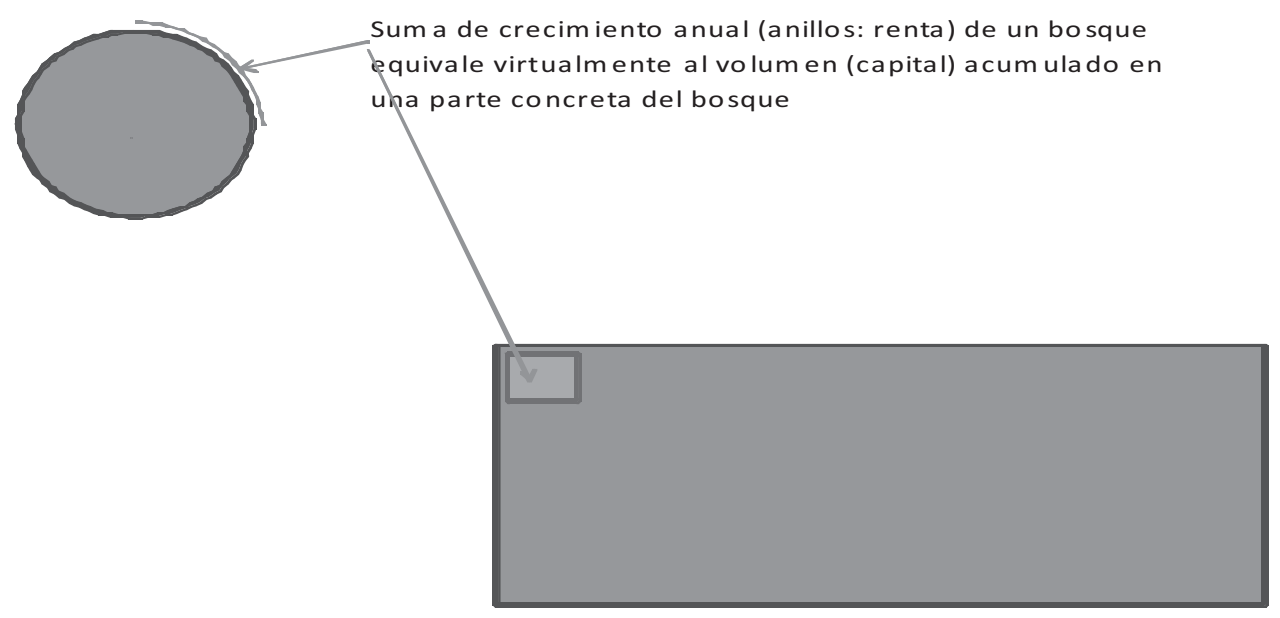

Figura 4. Concepto de equivalencia virtual de la persistencia forestal (Nachhaltigkeit) definido por Carlowitz

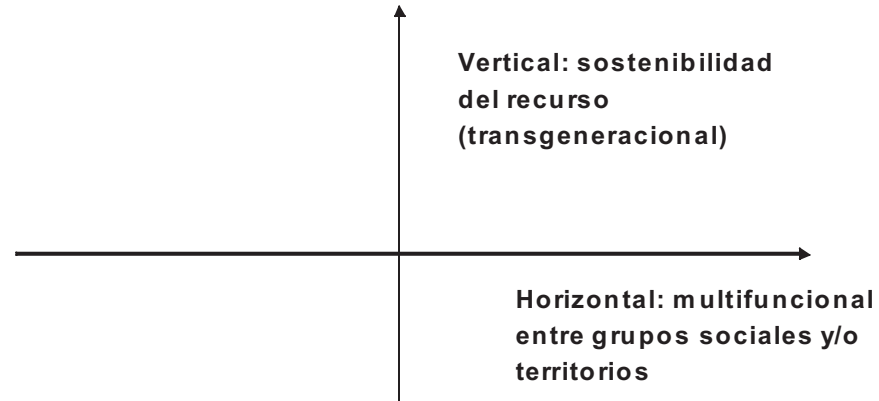

amplian el eje vertical con otro horizontal que focaliza en la necesaria solidad intersocial e interterritorial (Figura 5).

En definitiva, la gestión forestal multifuncional constituye una avanzadilla en la integración de los tres pilares de la sostenibilidad y un acervo y patrimonio cultural de sostenibilidad aplicada durante un considerable espacio de tiempo. Solo este hecho justificaría de por sí su preservación como fuente de know how, inspiración y experiencia.

\section{TEMAS EMERGENTES}

Entre los temas emergentes destacarían los bosques semi-naturales y los secos, el régimen
Figura 5. Dos ejes del principio de sostenibilidad

internacional de los bosques, la mejora de la gobernanza forestal y la comunicación forestal.

\section{Los bosques semi-naturales}

$62 \%$ de los bosques del planeta corresponden a bosques semi-naturales, en Europa 95\%. Pese a ello, el debate forestal internacional está muy centrado en las otras dos categorías (bosques naturales o vírgenes que ocupan un $36 \%$ ) y plantaciones intensivas que ocupan un $2 \%$. Con frecuencia son infravalorados al calificarse de bosques degradados, confundiendo el estado de una parte de ellos con la totalidad o su potencial. Por un lado son aquellos donde existe un mayor margen de sinergia y de gestión innovadora y además están ubicados en zonas accesibles y habitadas. Se olvida 
también que tanto la restauración forestal como el abandono rural aumentará su porcentaje de forma considerable. Finalmente cabe señalar que el término bosques plantados establecido hace un decenio es muy confuso dado que asemeja esa categoría con las plantaciones intensivas obviando que la mayoría de ellos (314) son repoblaciones protectoras o multifuncionales cuyo destino es disolverse entre los bosques semi-naturales.

\section{Los bosques secos}

Durante las últimas décadas se ha prestado mucha atención a los bosques tropicales húmedos pero relativamente poca a los secos que ocupan una considerable extensión (30\%) si bien son menos espectaculares. Los bosques secos están estrechamente relacionados con zonas social y políticamente inestables, se trata de las zonas más amenazadas por el cambio climático, y con un mayor crecimiento demográfico. También cabe reconocer que la Convención de lucha contra la desertificación (UNCCD) es la más débil de las tres convenciones de Río. También es necesario constatar que los aspectos claves en estas zonas, el suelo y el agua carecen de portavoces y reciben, pese a su condición de recursos básicos para la vida, muy poca atención social (interés afónico). En muchos casos, su degradación es de causa histórica que aunque haya cesado, es muy improbable su recuperación espontánea en plazos de tiempo socialmente aceptables. En definitiva, es en estas zonas donde se encuentra el bloque de áreas a restaurar y donde su efecto beneficioso será más palpable y su restauración poco conflictiva.

\section{El régimen internacional de los bosques}

La ubicación de los bosques en el interior de los países, su tradicional función de suministro de madera y su propiedad predominantemente pública explica su tardía inclusión en el derecho internacional (Pons, 2004). La Cumbre de Río de 1992 fracasó respecto de la $4^{\mathrm{a}}$ convención (forestal) estableciéndose en 2000 el Foro Foresta de la ONU (UNFF) con el objetivo de mantener la llama encendida. En 2015 se revisará UNFF incluyendo entre las opciones retomar una posible convención global forestal. Europa lanzó en 2011 un proceso regional con el objetivo de volver a retomar este proceso a esa escala que deberá culminarse en la Conferencia
Ministerial de Forest Europe prevista en Madrid en 2015 (INC, 2013).

\section{La mejora de la gobernanza forestal}

La participación es un elemento consustancial de la gobernanza moderna, y muy especialmente de los recursos naturales. Su función es complementaria de los sistemas representativos, especialmente cuando las decisiones políticas afectan de sobremanera a una parte de la población. Para ser efectiva resulta clave un enfoque honesto, comprometido con la participación a largo plazo, inclusivo y donde las respectivas responsabilidades estén claras en todo momento dado que las responsabilidades de gobierno no son diluibles. En este contexto, el rol de la sociedad civil forestal es fundamental y su madurez y complejidad es un termómetro y garantía de gobernanzas sólidas y su correspondiente resiliencia mientras que los poderes públicos se limitan cada vez más a establecer un marco y el seguimiento de la legislación.

\section{La comunicación forestal}

El proceso de urbanización afecta a todas las regiones lo que comporta que las poblaciones sean cada vez más urbanas y por ello pierdan sus originales raíces rurales (Figura 6).

Debe recordarse que aunque la imagen del bosque es ideal como elemento de comunicación, las operaciones forestales (corta, caza) se realizan en el patio trasero de la población urbana que ve el bosque desde el prisma de un elemento terciario (oasis, pulmón, vergel) y que fácilmente los malinterpreta como un atentado ecológico. Esta percepción no es casualidad sino que numerosas campañas bienintencionadas a diversas escalas la han venido reforzando. Por ello, esta percepción solo se podrá contrarrestar mediante campañas de comunicación forestal decididas, proactivas y permanentes donde la sociedad civil forestal resulta clave. Se hace necesario superar la asociación de los bosques con noticias negativas (incendios) pese a que paradójicamente sus servicios son ampliamente positivos para la sociedad.

Se trata de un ejemplo más que evidencia cual necesario es desarrollar el tercer pilar social de lo forestal que deviene clave para desactivar maniqueos conflictos entre el enfoque económi- 


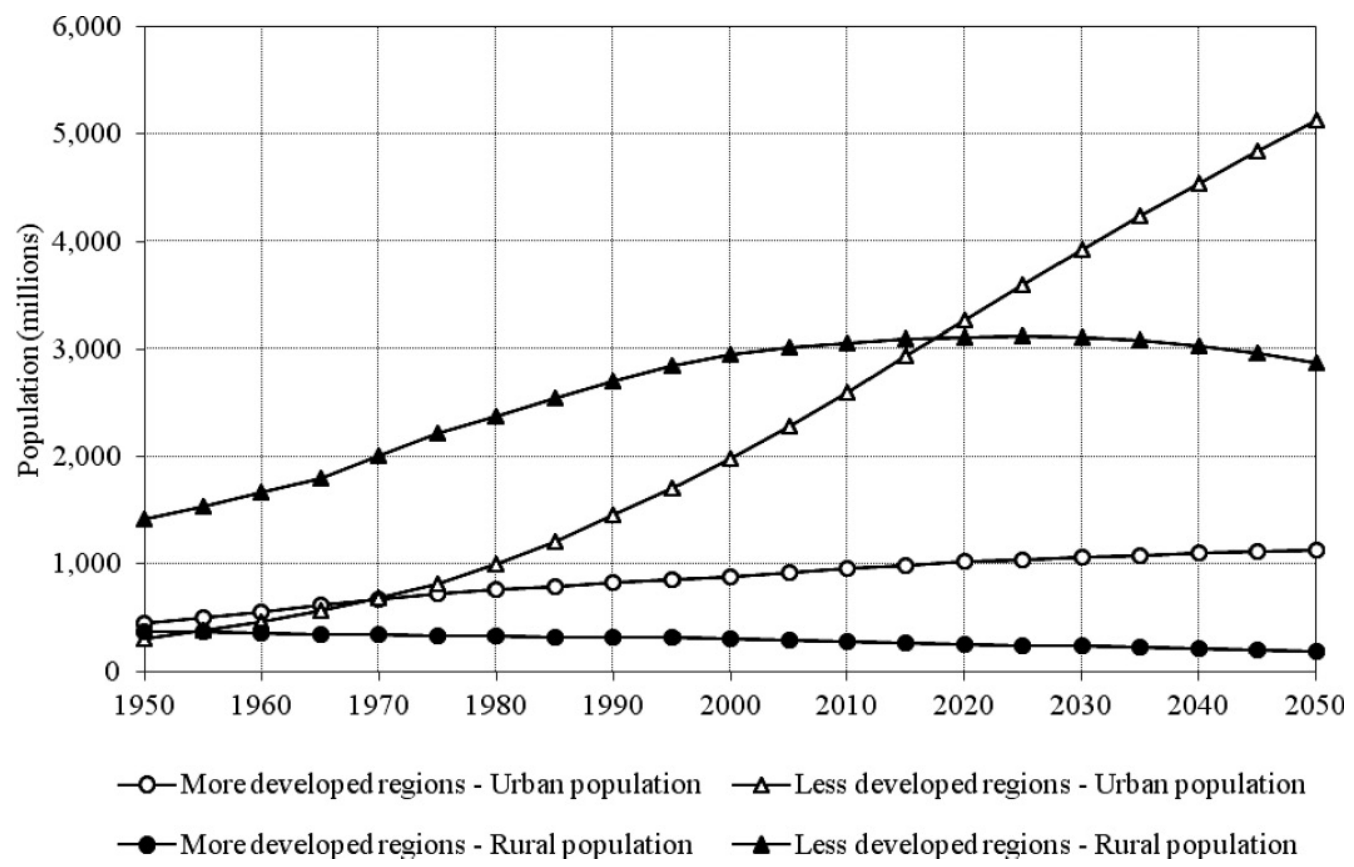

Figura 6. Evolución prevista de la población por bloques de desarrollo y medio urbano o rural (ONU, 2012b)

co (aprovechamiento) y ambiental (preservación en pro de la biodiversidad) pese a las débiles evidencias de su incompatibilidad.

Se hace necesario utilizar los mecanismos emergentes como las redes sociales y las ocasiones como el Año Internacional de los Bosques 2011 o el Día Internacional de los Bosques establecido desde 2013 oficialmente en cada 21 marzo ofrecen.

\section{CONCLUSIONES: LAS OPORTUNIDADES DE 2015}

El año 2015 alberga multitud de oportunidades para abordar los retos planteados. La aprobación de la agenda de la ONU post-2015 y los correspondientes objetivos de desarrollo sostenible, el acuerdo final de cambio climático de Paris, el XIV Congreso Forestal Mundial (Durban, Sudáfrica), la Expo 2015 Milán dedicada a la alimentación, la revisión del Foro Forestal de la ONU, el Año Internacional de los Suelos o la celebración de Beijing+20 y con ello el refuerzo de las políticas de igualdad de género constitu- yen excelentes oportunidades para ello. En qué medida sean capaces los actores forestales de visualizar el rol de los bosques en la resolución retos complejos planteados actualmente a la Humanidad será finalmente determinante de su relevancia y visibilidad a escala global. Todo un reto de comunicación.

\section{BIBLIOGRAFÍA}

CARlowitz Von, H.C.; 1713. Sylvicultura Oeconomica oder Hausswirthliche Nachricht und Naturgemäße Anweisung zur Wilden Baum-Zucht. Leibzig. (Reprint 2nd edition, 2009, Remagen-Oberwinter, Verlag Kessel).

FAO; 2010. Evaluación de los recursos forestales mundiales 2010. Forestry Papers 163. Roma.

FAO; 2012a. World agriculture towards 2030/2050: the 2012 revision. ESA Working paper 12-03: 1-147.

FAO; 2012b. Directrices voluntarias sobre la gobernanza responsable de la tenencia de la tierra, la 
pesca y los bosques en el contexto de la seguridad alimentaria nacional. FAO. Roma.

FAO; 2013. Improving governance of forest tenure. Roma.

FAO; 2014. FAO Food Price Index. Roma. http://www.fao.org/worldfoodsituation/food pricesindex/en/.

GPFLR; 2014. Global forest map of forest landscape restoration opportunities. Global Partnership on Forest Landscape Restoration. $\mathrm{http} / / / \mathrm{www}$. forestlandscaperestoration.org/top ic/map-and-analyse-restoration-potential.

INC; 2013. Legally binding agreement on forests in Europe. http://www.forestnegotiations.org/.

ONU; 1987. Our common future. Report of the World Commission on Environment and Development. New York.

ONU; 2012. El futuro que queremos. DESA. New York. http://www.uncsd2012.org/con- tent/documents/778futurewewant_spanish.pdf.

ONU; 2012b. World Urbanization Prospects. The 2011 Revision. DESA, New York, http://esa.un.org/unup/pdf/WUP2011_Highl ights.pdf: 3 .

ONU; 2014.

http://sustainabledevelopment.un.org/owg.html

Pons, X.; 2004. El régimen forestal internacional. Monografías INIA Forestal 6. Madrid.

RoJAS, E.; 2011. Los poderes locales y el patrimonio forestal. Carta Local 241: 52-53.

Rojas, E.; 2014. De Carlowitz a la Cumbre Río+20: 300 años de evolución del principio de sostenibilidad. RSEAP, Anales 2013. Valencia (en imprenta).

WEIZSÄCKER, E.U. vON; 1997. Nachhaltigkeit ein forstliches Prinzip wird neu entdeckt. Jahresbericht 1996: 37-48. Deutscher Forstverein. Niedenstein. 